PROCEEDINGS OF THE

AMERICAN MATHEMATICAL SOCIETY

Volume 130, Number 12, Pages 3515-3518

S 0002-9939(02)06518-8

Article electronically published on May 1, 2002

\title{
INTEGRAL REPRESENTATIONS FOR THE ALTERNATING GROUPS
}

\author{
UDO RIESE
}

(Communicated by Stephen D. Smith)

\begin{abstract}
We show that every complex representation of an alternating group can be realized over the ring of integers of a "small" abelian number field.
\end{abstract}

\section{INTRODUCTION}

By a well-known theorem of R. Brauer every (irreducible) complex character $\chi$ of a finite group $G$ can be written in the $g$-th cyclotomic field $\mathbb{Q}\left(\zeta_{g}\right)$ where $g$ denotes the exponent of $G$. It remains a problem as to whether this can be done integrally, i.e. if there exists a matrix representation with entries in the ring of integers $\mathbb{Z}\left[\zeta_{g}\right]$ affording $\chi$.

For solvable groups $G$ this was shown to be true by Cliff, Ritter and Weiss 1 . In general, Clifford theory reduces the question to representations of quasi-simple groups (stable under some automorphisms) (Knapp-Schmid [4]). So far, integral representations for all irreducible characters of simple groups have been constructed for the sporadic groups, some small alternating groups, some groups of Lie-type of small order [4] and for the groups $\operatorname{SL}(2, p)$ over the prime field [6].

Theorem 1. Every irreducible complex representation of the alternating group $A_{n}$ can be realized over the ring of integers of the field

$$
\mathbb{Q}\left(\sqrt{p^{*}} \mid p \text { odd prime, } p \leq n\right),
$$

where $p^{*}=(-1)^{\frac{p-1}{2}} p$ for any odd prime $p$.

Obviously, the field given in the theorem is contained in the $g$-th cyclotomic field $\left(g=\exp \left(A_{n}\right)\right)$. The proof of the theorem is based on a capitulation theorem for ideal classes by Terada [8] which enables us to realize all characters by the same argument. Our method also gives partial results for the covering groups $\tilde{A}_{n}$ of the alternating groups but a complete answer for these groups seems to require a more specific approach.

\section{Ambiguous ideAl Classes}

Let $K / k$ be a cyclic Galois extension of an algebraic number field $k$. We denote by $\tau$ a generator of the Galois group. An ideal class $[I]$ in the (finite) class group

Received by the editors May 3, 2001 and, in revised form, July 30, 2001.

2000 Mathematics Subject Classification. Primary 20C10, 20C30.

(C)2002 American Mathematical Society 
$C_{K}$ of $K$ is called ambiguous if it is fixed by $\tau$, that is, if it is contained in the kernel of the homomorphism

$$
\phi: C_{K} \rightarrow C_{K}:[I] \mapsto[I]^{1-\tau} .
$$

The image of $\phi$ is the principal genus; the genus field $K_{\Gamma}$ of $K / k$ is the class field corresponding to the principal genus. An ideal $I$ in an ambiguous class $[I]$ is an ambiguous class ideal. It is itself ambiguous if $I^{1-\tau}=(1)$. Note that in general not every ambiguous class can be represented by ambiguous ideals, the latter might form a subgroup of index 2 in the group of ambigous class ideals.

Proposition 1 (Terada [8]). Every ambiguous ideal class of a cyclic Galois extension $K / k$ becomes trivial in the genus field $K_{\Gamma}$ of $K / k$.

\section{Lattices over DedeKind domains}

Let $K$ be an algebraic number field and let $R=R_{K}$ be the ring of algebraic integers in $K$. Let $G$ be a finite group and $W$ an absolutely irreducible $K G$-module affording the character $\chi$. There exists a (torsion-free, finitely generated) $R G$ lattice $U$ in $W$ such that $W=K U \cong K \otimes_{R} U$ (by taking all $R$-linear combinations of the $G$-images of a $K$-basis of $W$ ). Every $R G$-lattice of this type is isomorphic to such a full $R G$-lattice in $W$. We wish to find a lattice which is $R$-free (not just $R$-projective).

Let $U$ be a full $R G$-lattice in $W$. As an $R$-module $U$ is the direct sum of $s=\chi(1)$ (nonzero) ideals $J_{i}$ of $R$. By a well known theorem of Steinitz this rank $s$ together with the Steinitz class

$$
[U]=\left[\prod_{i} J_{i}\right]=\prod_{i}\left[J_{i}\right] \in C_{K}
$$

in the class group of the Dedekind domain $R$ determine the $R$-isomorphism type of $U$. The genus $\gamma(U)$ of $U$ consists, in the present situation, of all $R G$-lattices of the form $J U$ for some fractional ideal $J$ of $R$. We have

$$
[J U]=[J]^{s}[U],
$$

and there are exactly $\left|C_{K}\right|$ different isomorphism types of $R G$-lattices in $\gamma(U)$ (see [2], (31.26)).

\section{Proof of the theorem}

Every irreducible (complex) character of a symmetric group $S_{n}$ has trivial Schurindex over the rationals (cf. [3], Theorem 2.1.12). Furthermore, every character of $S_{n}$ has its values in $\mathbb{Q}$, thus every irreducible character of an alternating group has trivial Schur-index over the rationals (cf. [7, Example 3). In particular, there are integral representaions over the principal ideal ring $\mathbb{Z}$ for all irreducible characters of $S_{n}$.

Lemma 1. Let $\chi \in \operatorname{Irr}\left(A_{n}\right)$ be an irreducible (complex) character. Then either $\mathbb{Q}(\chi)=\mathbb{Q}$ or $\mathbb{Q}(\chi)=\mathbb{Q}(\sqrt{d})$ for some odd square-free integer $d \neq 1$ with $d \equiv 1$ $(\bmod 4)$.

Proof. By [3], Theorem 2.5.13, either $\mathbb{Q}(\chi)=\mathbb{Q}$ or $\mathbb{Q}(\chi)$ is a quadratic number

field. In fact, if $\mathbb{Q}(\chi) \neq \mathbb{Q}$, then $\chi$ belongs to a self-associated partition $\alpha$ of $n$ 
(with diagram $[\alpha]$ symmetric with respect to the main diagonal). Furthermore, for $g \in A_{n}$ either $\chi(g)$ is an integer or

$$
\chi(g)=\frac{1}{2}\left(\varepsilon \pm \sqrt{\varepsilon \prod_{i} h_{i i}^{\alpha}}\right),
$$

where $\varepsilon=(-1)^{\frac{n-k}{2}}, k$ being the length of the main diagonal of the diagram $[\alpha]$, and where the $h_{i i}^{\alpha}$ are the lengths of the main hooks of $[\alpha]$. All the hook lengths $h_{i i}^{\alpha}$ are odd. Now use that $\chi(g)$ is an algebraic integer, and use that odd squares are congruent to $1 \bmod 4$.

Observe that in the lemma $d$ is the (absolute) discriminant of the quadratic number field $K=\mathbb{Q}(\sqrt{d})$ and that $d \leq n$ !. We have a unique *-decomposition (see Leopoldt [5] or Terada [8, Section 2)

$$
d=\prod p^{*}
$$

where $p^{*}=(-1)^{\frac{p-1}{2}} p$ and the product is taken over all (odd) prime divisors $p$ of $d$ (so that $p \leq n$ ). The field

$$
K^{*}=\mathbb{Q}\left(\sqrt{p^{*}} \mid p \text { divides } d\right)
$$

is the so-called genus field in the narrow sense which always contains the genus field $K_{\Gamma}$ of $K / \mathbb{Q}$ with degree $\left[K^{*}: K_{\Gamma}\right] \leq 2$. It follows from Terada's theorem that every ambiguous ideal class in $C_{K}$ becomes trivial in $C_{K^{*}}$.

By Clifford the restriction to $A_{n}$ of an irreducible character of $S_{n}$ remains irreducible or decomposes into the sum of two conjugate irreducibles. Since all Schur indices of the characters involved are trivial, the rational valued characters of $A_{n}$ are realizable over the integers (as every torsion-free, finitely generated $\mathbb{Z}$-module is free). The theorem thus is an immediate consequence of the following:

Proposition 2. Let $\chi$ be an irreducible character of $A_{n}$ such that $\mathbb{Q}(\chi)=\mathbb{Q}(\sqrt{d}) \neq$ $\mathbb{Q}$ where $d$ is a (odd) square-free integer. Let $R^{*}$ be the ring of algebraic integers in

$$
K^{*}=\mathbb{Q}\left(\sqrt{p^{*}} \mid p \text { divides } d\right) .
$$

Then there is a $R^{*}$-free $R^{*} A_{n}$-module $V$ affording the character $\chi$.

Proof. We know that $d \equiv 1(\bmod 4)$ is odd (Lemma 1$)$. Let $W$ be an absolutely irreducible $\mathbb{Q} S_{n}$-module affording the (irreducible) character $\chi^{S_{n}}$. Then $W$, as a $\mathbb{Q} A_{n}$-module, is irreducible but not absolutely irreducible. In fact, since the Schur index $\mathrm{m}(\chi)=1$,

$$
K=\operatorname{End}_{\mathbb{Q} A_{n}}(W)
$$

is a quadratic number field with discriminant $d=p_{1}^{*} \cdots p_{t}^{*}$. Thus $K \cong \mathbb{Q}(\chi) \subseteq \mathbb{C}$ and we choose this isomorphism in such a way that $W$, regarded as an absolutely irreducible (right) $K A_{n}$-module, affords the character $\chi$.

Let $R$ be the integral closure of $\mathbb{Z}$ in $K$, and let $U \subseteq W$ be a full (right) $R A_{n^{-}}$ lattice. Then

$$
\operatorname{End}_{R A_{n}}(U)=R \text {. }
$$

Let $\tau$ denote the element of $\operatorname{End}_{\mathbb{Q}}(W)$ induced by the transposition (12). Then $\tau$ induces the non-trivial automorphism on $K$ which we denote by $\tau$ as well. 
Both $U$ and $U \tau$ are (right) full $R A_{n}$-lattices in the $K A_{n}$-module $W$. Suppose $\left\{w_{i}\right\}_{i=1}^{s}$ is a $K$-basis of $W$ (so that $\left.s=\chi(1)\right)$. Then

$$
U=\bigoplus_{i=1}^{s} w_{i} J_{i}
$$

for certain fractional ideals $J_{i}$ of $R$. Now $\left\{w_{i} \tau\right\}_{i=1}^{s}$ is also a $K$-basis of $W$, and

$$
U \tau=\bigoplus_{i=1}^{s}\left(w_{i} J_{i}\right) \tau=\bigoplus_{i=1}^{s} w_{i} \tau J_{i}^{\tau}
$$

Consequently the Steinitz class $[U \tau]=\left[\Pi J_{i}^{\tau}\right]=[U]^{\tau}$ where $\tau$ acts naturally on the class group $C_{K}$ (by inversion, $[U]^{\tau}=[U]^{-1}$ ).

Now put $V=U+U \tau$. This is again a full $R A_{n}$-lattice in $W$ and as $\tau^{2}=1$ it is $\tau$-invariant. Thus $[V]=[V]^{\tau}$ is an ambiguous ideal class in $C_{K}$. By Terada's theorem (Proposition 1), and since $K_{\Gamma} \subseteq K^{*}$, the ideal class $[V]$ becomes trivial in $C_{K^{*}}$, that is, it is in the kernel of the natural map $C_{K} \rightarrow C_{K^{*}}$. Here for convenience we identify $K=\mathbb{Q}(\sqrt{d})$. Now $W^{*}=K^{*} \otimes_{K} W$ is an absolutely irreducible $K^{*} A_{n^{-}}$ module affording $\chi$, and $V^{*}=R^{*} \otimes_{R} V$ is a full $R^{*} A_{n}$-lattice in $W^{*}$. The Steinitz class $\left[V^{*}\right] \in C_{K^{*}}$ is the image of $[V]$ under the natural map. Thus $V^{*}$ is $R^{*}$-free, as desired.

\section{REFERENCES}

1. G. Cliff, J. Ritter and A. Weiss, Group representations and integrality, J. Reine Angew. Math. 426 (1992), 193-202. MR 93f:20011

2. C.W. Curtis and I. Reiner, Methods of representation theory, I, Wiley, New York, 1981. MR 82i:20001 reprint MR 90k:20001

3. G. James and A. Kerber, The representation theory of the symmetric groups, Addison-Wesley, London, 1981. MR 83k:20003

4. W. Knapp and P. Schmid, An extension theorem for integral representations, J. Austral. Math. Soc. (Series A) 63 (1997), 1-15. MR 98m:20014

5. H. Leopoldt, Zur Geschlechtertheorie in abelschen Zahlkörpern, Math. Nachrichten 9 (1953), 351-362. MR 15:14d

6. U. Riese, On integral representations for $S L(2, q)$, J. Algebra 242 (2001), 729-739.

7. U. Riese and P. Schmid, Schur indices and Schur groups, II, J. Algebra 182 (1996), 183-200. MR 97e:20009

8. F. Terada, A principal ideal theorem in the genus fields, Tôhoku Math. J. 23 (1971), 697-718. MR 46:5285

Universität Tübingen, Mathematisches Institut, Auf Der Morgenstelle 10, D-72076 Tübingen, Germany

E-mail address: udo.riese@uni-tuebingen.de 